\title{
Bit Error Rate Analysis in IEEE 802.15.3a UWB Channels
}

\author{
Li-Chun Wang, Senior Member, IEEE, and Wei-Cheng Liu, Member, IEEE
}

\begin{abstract}
In this paper, we present a computable bit error rate (BER) expression for the binary signals in the IEEE 802.15.3a ultra-wideband (UWB) channel. In the literature, the impacts of the RAKE receiver's finger numbers and lognormal shadowing on the BER performance have not been reported yet. We propose a characteristic function (CF) based BER formula to overcome the convergence problem of the existing moment generating function (MGF) approach when the BER calculation takes account of the shadowing effect. Simulation results demonstrate that the proposed CF-based computable BER formula can accurately estimate the complete effects of the cluster and ray arrival processes, the lognormal fading as well as shadowing, and the finger numbers at RAKE receivers.
\end{abstract}

Index Terms-Bit error rate (BER), IEEE 802.15.3a channel model, RAKE receiver, shadowing, ultra-wideband (UWB).

\section{INTRODUCTION}

$\mathbf{U}$ LTRA-WIDEBAND (UWB) is a promising wireless communications technique for high data rate transmission. The UWB channel characteristics are very different from conventional narrowband channels. Recently, the IEEE 802.15.3a UWB channel model [1] is specified and widely adopted in the industry. However, UWB systems based on the IEEE 802.15.3a model are only evaluated by simulations or by analysis with simplified conditions.

\section{A. Problem Statement}

Hence, a fundamental question arises: how can a UWB system be analyzed in the complete IEEE 802.15.3a channel model? This UWB channel has two significant properties. First, because the UWB signal bandwidth is much wider than the channel coherence bandwidth, highly frequency selective fading exists. Second, UWB signals usually yield many clusters of non-Rayleigh faded rays because the extremely large bandwidth leads to high-resolution arrival time after being reflected by objects. The challenges of analyzing UWB signals in the IEEE 802.15.3a channel can be summarized into two types:

- Instead of fixed-number arrival rays within one cluster for narrowband channel, the UWB signal may arrive in

Manuscript received April 25, 2008; revised March 30. 2009, August 25, 2009, and November 22, 2009, accepted January 21, 2010. The associate editor coordinating the review of this paper and approving it for publication was L. Yang.

This work was supported by the National Science Council, Taiwan, under the contract NSC94-2213-E-009-030.

L.-C. Wang is with the Department of Communications Engineering, National Chiao Tung University, Hsinchu, Taiwan (e-mail: lichun@cc.nctu.edu.tw).

W.-C. Liu is with the Department of Communications Engineering, National Chung Cheng University, Chia-Yi, Taiwan (e-mail: comwcliu@ccu.edu.tw).

Digital Object Identifier 10.1109/TWC.2010.080571 many clusters with a random number of rays. The arrival processes of clusters and rays are modeled by a doubly stochastic Poisson process in the IEEE 802.15.3a model. Due to the unknown numbers of rays and clusters, it is difficult to compute the total collected signal energy at RAKE receivers.

- The multipath fading in UWB channels is not modeled as a traditional Rayleigh random variable because the central limit theorem is not applicable for insufficient arrival rays in a very narrow time bin. From measurement results [2], [3], a lognormal multipath fading as well as shadowing is adopted in the IEEE 802.15.3a UWB channel. Conditioned on the given number of rays as well as clusters, and the average amplitude, a UWB signal amplitude in the IEEE 802.15.3a channel is modeled as a two-dimensional lognormal random variable. Because the mean of signal amplitude is related to the Erlangdistributed inter-arrival time for clusters and rays, such a random signal is difficult to be analyzed.

\section{B. Related Work}

In the literature the current research related to the performance analysis of UWB systems can be categorized into two folds. Firstly, the UWB system has been investigated based on simpler channel models [4]-[7]. The channel models in [4]-[7] do not consider the cluster phenomenon and shadowing effect as in the IEEE 802.15.3a channel model and therefore are insufficient and unrealistic for performance analysis compared to our proposed method. Secondly, [8]-[13] investigated the performance of UWB systems based on more sophisticated UWB channels, such as the IEEE 802.15.3a model. In [8], the authors applied the techniques of counting integrals and shot noise to derive the computation BER formula in the IEEE 802.15.3a channel assuming the received waveform can be observed over a finite-length window. In [9], the output SNR statistics at the RAKE receiver in the IEEE 802.15.3a channel was presented, but the explicit BER formula for RAKE receivers taking account of shadowing was not presented. [10] analyzed the pairwise error probability (PEP) and outage probability of multiband orthogonal frequency-division multiplexing (OFDM) systems in the IEEE 802.15.3a channel model, but ignored the effect of the lognormal shadowing. [11] analyzed the effect of multiple antennas on the UWB system under a generalized UWB channel. In [12], the error performance of a multi-antenna RAKE receiver was analyzed over the frequency-selective UWB lognormal fading channels. [13] analyzed the signal-to-interference-plus-noise ratio (SINR) of direct sequence (DS) UWB systems in generalized SalehValenzuela channels based on the theory of renewal process. However, the method in [13] did not provide the BER formula 
and did not consider the shadowing effect. Compared with [11]-[13], the computational BER formula provided in this paper considers the complete IEEE 802.15.3a UWB channels with shadowing and the number of RAKE receiver fingers taken into account.

\section{Contribution and Organization of This Paper}

The contribution of this paper is developing an analytical method to compute the error performance in the complete IEEE 802.15.3a channel. We will present an explicit BER analytical computation method incorporating the impacts of the number of fingers at the RAKE receiver, the effects of shadowing and all the UWB channel parameters based on the IEEE 802.15.3a model. The proposed computable formula can easily analyze the effects of various UWB channel parameters in the IEEE 802.15.3a channel model without time consuming simulations. The calculations of BER can be performed by (11) with all required parameters and functions substituted in, which will be described in Section II.

The rest of this paper is organized as follows. In Section II, we derive the BER computable formula of UWB signals in the IEEE 802.15.3a channel. Section III presents the numerical results. Finally, we give our conclusions in Section IV.

\section{BER ANALYSIS}

\section{A. Problem Formulation}

From [14] we know that the error probability of binary signals with the coherent RAKE receiver is $P_{2}\left(\gamma_{b}\right)=$ $Q\left(\sqrt{\gamma_{b}\left(1-\rho_{r}\right)}\right)$, where $\gamma_{b}$ is the received SNR and $\rho_{r}=$ -1 and $\rho_{r}=0$ for antipodal signals and orthogonal signals, respectively. Define $E_{\mathrm{b}}$ as the bit energy and $N_{0}$ the noise power spectrum density. Then, we can express the BER of the RAKE receiver as

$$
\begin{aligned}
& P_{2}=\mathbb{E}_{\mathcal{E}}\left[Q\left(\sqrt{\left(1-\rho_{r}\right) \frac{E_{\mathrm{b}}}{N_{0}} \mathcal{E}}\right)\right] \\
& =\int_{0}^{\infty} Q\left(\sqrt{\left(1-\rho_{r}\right) \frac{E_{\mathrm{b}}}{N_{0}} x}\right) f_{\mathcal{E}}(x) d x,
\end{aligned}
$$

where $f_{\mathcal{E}}(x)$ is the PDF of the received energy $\mathcal{E}$. The next step is finding $f_{\mathcal{E}}(x)$ in the IEEE 802.15.3a UWB channel.

\section{B. PDF of the Received Energy $(\mathcal{E})$}

In this subsection, we suggest an approximation technique for calculating $f_{\mathcal{E}}(x)$, which can facilitate the derivation of a computable BER formula taking account of all the UWB channel effects of the IEEE 802.15.3a model and the number of fingers of the RAKE receiver $(L)$. First, we conjecture that $\mathcal{E}$ is close to $\tilde{\mathcal{E}}$, the sum of squared signal amplitudes at each individual ray within a time window $\left[0, L T_{\mathrm{c}}\right]$, where $T_{\mathrm{c}}$ is the chip duration between two consecutive fingers. Then $\tilde{\mathcal{E}}$ can approximate the signal energy collected by the $L$ fingers of the RAKE receiver. That is,

$$
\tilde{\mathcal{E}}=X^{2} \sum_{(k, l): 0 \leq T_{l}+\tau_{k, l} \leq L T_{\mathrm{c}}} \alpha_{k, l}^{2}=X^{2}\left(\alpha_{0,0}^{2}+\tilde{\Phi}_{r 0}+\tilde{\Phi}_{\otimes}\right),
$$

where $X$ is the lognormal shadowing random variable, $\alpha_{k, l}$ is the amplitude of the $k$ th ray in the $l$ th cluster and has lognormal distribution, $\tilde{\Phi}_{r 0}$ is the sum of squared path gains of the first cluster excluding $\alpha_{0,0}^{2}$, and $\tilde{\Phi}_{\otimes}$ is the sum of squared path gains of rays in the remaining clusters. Note that $X^{2}, \alpha_{0,0}^{2}, \tilde{\Phi}_{r 0}$, and $\tilde{\Phi}_{\otimes}$ are statistically independent. Denote the received energy without shadowing by $\tilde{\mathcal{E}}_{X 0} \triangleq \alpha_{0,0}^{2}+\tilde{\Phi}_{r 0}+$ $\tilde{\Phi}_{\otimes}$. The three independent terms of the decomposition reflect the corresponding decomposition of the counting measure on the two-dimensional Poisson process behind the IEEE 802.15.3a channel model [15]. The characteristic function $\Psi_{\tilde{\mathcal{E}}_{X 0}}(\nu)$ of $\tilde{\mathcal{E}}_{X 0}$ in the IEEE 802 .15.3a UWB channel can be written as

$$
\Psi_{\tilde{\mathcal{E}}_{X 0}}(\nu)=\tilde{\mathcal{L}}_{0,0}(\nu) \tilde{R}(\nu, L) \tilde{S}(\nu, L),
$$

where $\tilde{\mathcal{L}}_{T, t}(\nu), \tilde{R}(\nu, L)$, and $\tilde{S}(\nu, L)$ are the characteristic functions of $\alpha_{T, t}^{2}, \tilde{\Phi}_{r 0}$, and $\tilde{\Phi}_{\otimes}$, respectively. Here $\alpha_{T, t}$ denotes the signal amplitude at a path arriving at time $t$ in a cluster arriving at time $T$. Compared with [15], we further consider the effects of $X, L$, and $T_{\mathrm{c}}$. The following theorem gives the computation formula of $\tilde{\mathcal{L}}_{T, t}(\nu)$.

Theorem 1: The characteristic function $\tilde{\mathcal{L}}_{T, t}(\nu)$ can be computed by

$$
\tilde{\mathcal{L}}_{T, t}(\nu)=\int_{0}^{\infty} e^{j \nu x} \frac{10 \exp \left[-\frac{1}{2 \sigma^{2}}\left(10 \log _{10} x-\mu_{T, t}\right)^{2}\right]}{\sqrt{2 \pi} \sigma x \ln 10} d x,
$$

where $\mu_{T, t}=\frac{10}{\ln 10}\left[\ln \Omega_{0}-\frac{T}{\Gamma}-\frac{t-T}{\gamma}-\left(\frac{\ln 10}{10}\right)^{2} \frac{\sigma^{2}}{2}\right]$ and $\sigma=\sqrt{\sigma_{1}^{2}+\sigma_{2}^{2}}$. The parameters $\Omega_{0}, \Gamma$, and $\gamma$ are defined in the IEEE 802.15.3a channel model.

Proof: Please see Appendix A.

Theorem 2: For the RAKE receiver with $L$ fingers, the function $\tilde{R}(\nu, L)$ in (3) can be written as $\tilde{R}(\nu, L)=$ $\exp \left[-\lambda \tilde{\psi}_{\nu}(0, L)\right]$ and function $\tilde{\psi}_{\nu}(T, L)$ can be computed by

$$
\tilde{\psi}_{\nu}(T, L)= \begin{cases}\int_{T}^{L T_{\mathrm{c}}}\left[1-\tilde{\mathcal{L}}_{T, t}(\nu)\right] d t, & T \leq L T_{\mathrm{c}}, \\ 0, & T>L T_{\mathrm{c}} .\end{cases}
$$

Similarly, $\tilde{S}(\nu, L)$ can be computed by $\tilde{S}(\nu, L)=$ $\exp [-\Lambda \tilde{J}(\nu, L)]$, where

$$
\tilde{J}(\nu, L)=\int_{0}^{L T_{\mathrm{c}}}\left[1-\tilde{\mathcal{L}}_{T, T}(\nu) e^{-\lambda \tilde{\psi}_{\nu}(T, L)}\right] d T .
$$

Proof: Please see Appendix B.

Substituting (4) into (5) and (6), we can obtain $\tilde{R}(\nu, L)$ and $\tilde{S}(\nu, L)$, respectively. The characteristic function $\Psi_{\tilde{\mathcal{E}}_{X 0}}(\nu)$ can be easily obtained by using (3). The PDF of $\tilde{\mathcal{E}}_{X 0}$ can be computed as $f_{\tilde{\mathcal{E}}_{X 0}}(x)=\frac{1}{2 \pi} \int_{-\infty}^{\infty} \Psi_{\tilde{\mathcal{E}}_{X 0}}(\nu) e^{-j x \nu} d \nu$. Finally, with shadowing the PDF of $\tilde{\mathcal{E}}$ can be computed as

$$
f_{\tilde{\mathcal{E}}}(x)=\int_{-\infty}^{\infty} \frac{1}{|y|} f_{X^{2}}\left(\frac{x}{y}\right) f_{\tilde{\mathcal{E}}_{X 0}}(y) d y .
$$

\section{Computable Formula}

The Gauss-Hermite formula approximates the integration of a function $f(x)$ over $(-\infty, \infty)$ to a weighted sum. The Gauss-Legendre formula is suitable for the integration on the interval $[0, \infty)$. These two formulas are both good for 
approximating values of integrations. The difference between these two formulas is the integration interval. Based on this property, we use the Gauss-Hermite formula to approximate $\tilde{\mathcal{L}}_{T, t}(\nu)$ in $(8), f_{\tilde{\mathcal{E}}_{X 0}}(x)$, and $f_{\tilde{\mathcal{E}}}(x)$ in $(7)$. On the other hand, we use the Gauss-Legendre formula to approximate $\tilde{\psi}_{\nu}(T, L)$ in (9) and $\tilde{J}(\nu, L)$ in (10).

After some computations, we can obtain the Hermite computation form for $\tilde{\mathcal{L}}_{T, t}(\nu)$ as

$$
\begin{aligned}
\tilde{\mathcal{L}}_{T, t}(\nu) & =\frac{1}{\sqrt{\pi}} \int_{-\infty}^{\infty} e^{-y^{2}} e^{j \nu 10^{\left(\sqrt{2} \sigma y+\mu_{T, t}\right) / 10}} d y \\
& \approx \frac{1}{\sqrt{\pi}} \sum_{k=1}^{N^{(\mathrm{H})}} w_{k}^{(\mathrm{H})} e^{j \nu 10^{\left(\sqrt{2} \sigma x_{k}^{(\mathrm{H})}+\mu_{T, t}\right) / 10}} \\
& =\sum_{k=1}^{N^{(\mathrm{H})}} w_{k}^{(\mathrm{H})} e^{\left(x_{k}^{(\mathrm{H})}\right)^{2}} f\left(x_{k}^{(\mathrm{H})}\right),
\end{aligned}
$$

where $x_{k}^{(\mathrm{H})}$ is the $k$-th root of the Hermite polynomial of the $N^{(\mathrm{H})}$-th order, and $w_{k}^{(\mathrm{H})}$ is the corresponding weight of the root $x_{k}^{(\mathrm{H})}$ and $f(x)=\frac{1}{\sqrt{\pi}} \exp \left(j \nu 10^{\left(\sqrt{2} \sigma x+\mu_{T, t}\right) / 10}-x^{2}\right)$.

Next, we apply the Gauss-Legendre formula [16], [17] to obtain the characteristic functions $\tilde{R}(\nu, L)$ and $\tilde{S}(\nu, L)$. Thus, the function $\tilde{\psi}_{\nu}(T, L)$ in the exponent of $\tilde{R}(\nu, L)$ can be computed by

$$
\tilde{\psi}_{\nu}(T, L) \approx \begin{cases}\frac{L T_{\mathrm{c}}-T}{2} \sum_{p=1}^{N^{(\mathrm{L})}} w_{p}^{(\mathrm{L})}\left[1-\tilde{\mathcal{L}}_{T, t}(\nu)\right] \mid & \\ t=\frac{L T_{\mathrm{c}}-T}{2} x_{p}^{(\mathrm{L})}+\frac{L T_{\mathrm{c}}+T}{2} & T \leq L T_{\mathrm{c}}, \\ 0 & T>L T_{\mathrm{c}},\end{cases}
$$

where $\left\{w_{p}^{(\mathrm{L})}\right\},\left\{x_{p}^{(\mathrm{L})}\right\}$, and $N^{(\mathrm{L})}$ are the weights, abscissas, and number of points of the Gauss-Legendre formula, respectively. Similarly, the function $\tilde{J}(\nu, L)$ in the exponent of $\tilde{S}(\nu, L)$ can be computed by

$$
\begin{gathered}
\tilde{J}(\nu, L) \approx \frac{L T_{\mathrm{c}}}{2} \sum_{i=1}^{N^{(\mathrm{L})}} w_{i}^{(\mathrm{L})}\left[1-\tilde{\mathcal{L}}_{T, T}(\nu) e^{-\lambda \tilde{\psi}_{\nu}(T, L)}\right] \mid \\
T=\frac{L T_{\mathrm{c}}}{2} x_{i}^{(\mathrm{L})}+\frac{L T_{\mathrm{c}}}{2} .
\end{gathered}
$$

Combining (7), (8), (9), and (10), the BER of the RAKE receiver in the IEEE 802.15.3a UWB channel can be computed by

$$
\begin{aligned}
\tilde{P}_{2}= & \frac{1}{2 \pi^{3 / 2}} \sum_{k=1}^{N^{(\mathrm{H})}} \sum_{l=1}^{N^{(\mathrm{H})}} \sum_{m=1}^{N^{(\mathrm{H})}} w_{k}^{(\mathrm{H})} w_{l}^{(\mathrm{H})} w_{m}^{(\mathrm{H})} \\
& \frac{1}{|y|} \exp \left(j \nu 10 \frac{\sqrt{2} \sigma x_{l}^{(\mathrm{H})}+\mu_{0,0}}{10}\right) \\
& \exp \left(-\left.\frac{1}{2} \lambda L T_{\mathrm{c}} \sum_{p=1}^{N^{(\mathrm{L})}} w_{p}^{(\mathrm{L})}\left[1-\tilde{\mathcal{L}}_{0, t}(\nu)\right]\right|_{t=\frac{1}{2} L T_{\mathrm{c}}\left(x_{p}^{(\mathrm{L})}+1\right)}\right) \\
& \exp \left(-\frac{1}{2} \Lambda L T_{\mathrm{c}} \sum_{i=1}^{N^{(\mathrm{L})}} w_{i}^{(\mathrm{L})}\left[1-\tilde{\mathcal{L}}_{T, T}(\nu) e^{-\lambda \tilde{\psi}_{\nu}(T, L)}\right] \mid\right. \\
& \left.T=\frac{1}{2} L T_{\mathrm{c}}\left(x_{i}^{(\mathrm{L})}+1\right)-j \nu y+\nu^{2}+y^{2}\right)
\end{aligned}
$$

$$
\left.\int_{0}^{\infty} Q\left(\sqrt{\left(1-\rho_{r}\right) \frac{E_{\mathrm{b}}}{N_{0}} x}\right) f_{X^{2}}\left(\frac{x}{y}\right) d x\right|_{\nu=x_{k}^{(\mathrm{H})}, y=x_{m}^{(\mathrm{H})}}
$$

Importantly, one can see that (11) requires only two integrals in the last line including the integral for evaluating the $Q$ function. Other integrals are replaced by the summations based on the Hermite and the Legendre polynomial methods with the $N^{(\mathrm{H})}$-th and $N^{(\mathrm{L})}$-th orders.

\section{Discussion}

Comparing to [8], we further consider the effects of the RAKE receiver and shadowing into the BER formula. This extension is nontrivial because of the following two reasons. First, the calculation of the energy collected by the RAKE receiver is very complicated. The complexity is proportional to the number of fingers of the RAKE receiver. More specifically, we have to find the energy on each finger and find the total energy. It is much more difficult than just find the energy during a certain time window. We find an approximation method that can evaluate the BER with a very small error as we will show in the numerical results. Second, adding shadowing involves an additional lognormal random variable in our problem. This implies we need one more integral in (11). We have tried to use the Craig's formula as in [8] to include the shadowing term $X$ into the BER formula as

$$
\begin{aligned}
\tilde{P}_{2}^{\prime} & =\mathbb{E}\left[\frac{1}{2 \pi} \int_{-1}^{1} \frac{\exp \left[-\eta X^{2} \Phi\left(0, L T_{\mathrm{c}}\right) / 2 y^{2}\right]}{\sqrt{1-y^{2}}} d y\right] \\
& =\frac{1}{2 \pi} \int_{-1}^{1} \frac{\mathbb{E}\left[M_{\Phi\left(0, L T_{\mathrm{c}}\right)}\left(-\eta X^{2} / 2 y^{2}\right)\right]}{\sqrt{1-y^{2}}} d y,
\end{aligned}
$$

where $\Phi\left(0, L T_{\mathrm{c}}\right)=\sum_{(k, l): 0 \leq T_{l}+\tau_{k, l} \leq L T_{\mathrm{c}}} \alpha_{k, l}^{2} \quad$ and $M_{\Phi\left(0, L T_{\mathrm{c}}\right)}(\cdot)$ is the MGF of $\Phi\left(0, L T_{\mathrm{c}}\right)$. However, as it will be shown in the next section, we find that the analytical BER obtained from (12) has a large error compared with simulation results. Hence, we develop the characteristic function based BER formula with shadowing in the IEEE 802.15.3a model and show that the corresponding analytical BER is close to the simulation BER in the next section. Therefore, we believe that adding the shadowing effect into the BER calculation under the IEEE 802.15.3a UWB channel model is not a straightforward extension from the existing work.

\section{NUMERICAL RESUlts}

In the numerical results, we set $N^{(\mathrm{H})}=32$ and $N^{(\mathrm{L})}=16$. Fig. 1 shows the comparison of our BER based on (11) with those by the simplified methods presented in references [4][7]. In Fig. 1, there is shadowing effect with the standard deviation $\sigma_{x}=3 \mathrm{~dB}$. Compared with the simulation BER, the simplified methods presented in [4]-[7] underestimate the BER.

Fig. 2 shows the shadowing effect with the standard deviation $\sigma_{x}=3$ and $6 \mathrm{~dB}$ on the BER performance of a 10finger RAKE receiver in the IEEE 802.15.3a UWB channel CM3 based on (11). At BER is 0.02 , the required $E_{\mathrm{b}} / N_{0}$ for $\sigma_{x}=6 \mathrm{~dB}$ is $3 \mathrm{~dB}$ higher than that for $\sigma_{x}=3 \mathrm{~dB}$. 


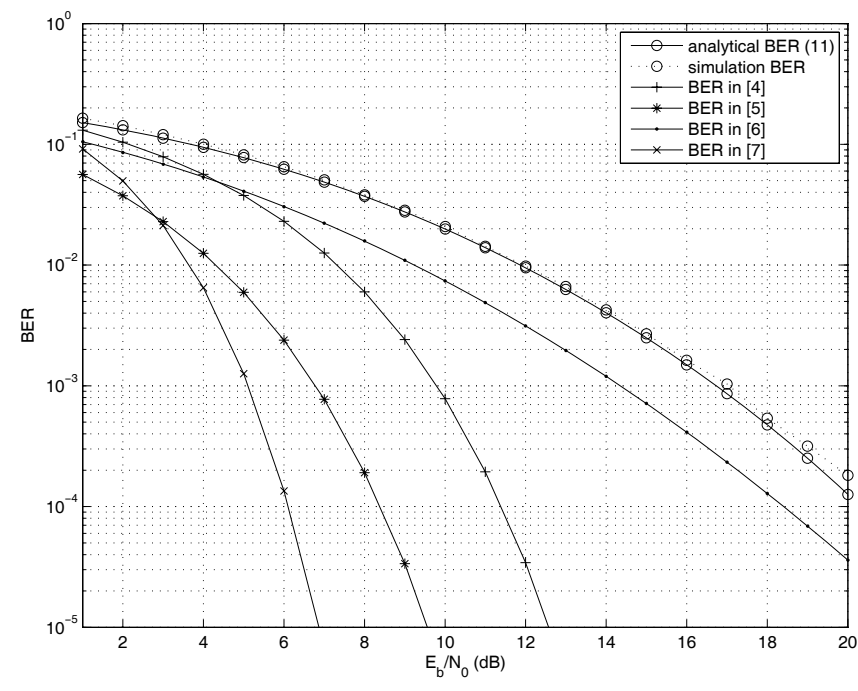

Fig. 1. Comparison of our BER based on (11) with those by the simplified methods presented in references [4]-[7]. The shadowing standard deviations $\sigma_{x}=3 \mathrm{~dB}$.

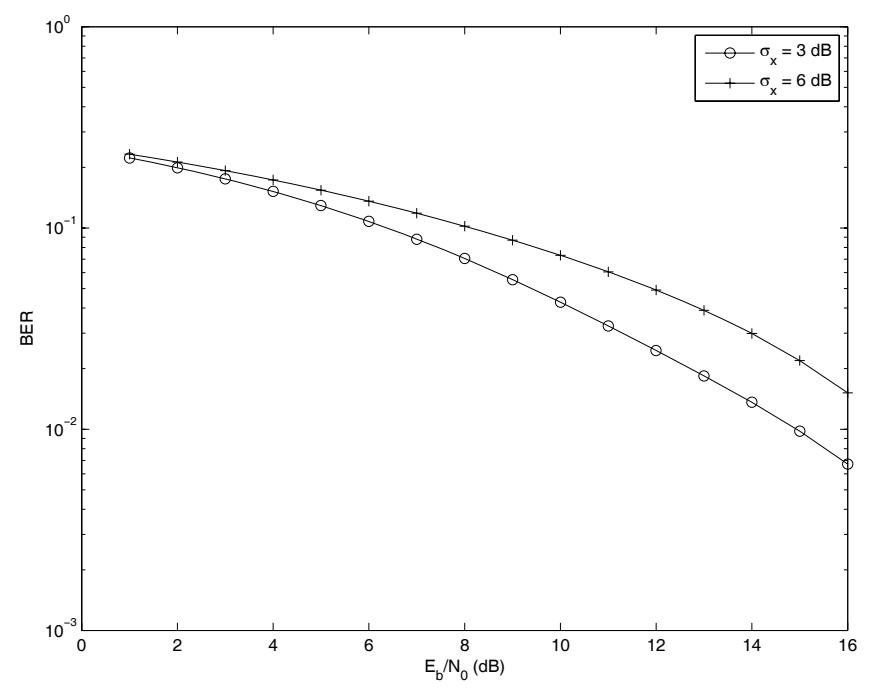

Fig. 2. Effect of various shadowing standard deviations $\left(\sigma_{x}=3 \mathrm{~dB}\right.$ and $6 \mathrm{~dB})$ on the BER performance of a 10-finger RAKE receiver in the IEEE 802.15.3a UWB channel CM3.

Hence, the shadowing effect in the IEEE 802.15.3a channel is quite significant and should not be ignored when evaluating the UWB system performance.

In Fig. 3, the proposed characteristic function (CF)-based analytical BER formula (11) is verified by simulations. As seen from Fig. 3, the analytical results according to (11) match the simulation results quite well in general. However, there are discrepancies between the simulation and the analytical curves at some points. This is because the usage of Gauss-Hermite and Gauss-Legendre formulas may cause some integration errors. Nevertheless, it is generally true that using $f_{\tilde{\mathcal{E}}}(x)$ can save a lot of calculation time compared with using $f_{\mathcal{E}}(x)$, and provide very good approximation to the simulative BER in the IEEE 802.15.3a UWB channel.

In Fig. 4, the MGF-based analytical BER formula (12) is examined by simulations for a 10-finger RAKE receiver in the IEEE 802.15.3a CM1 4 channels with shadowing standard

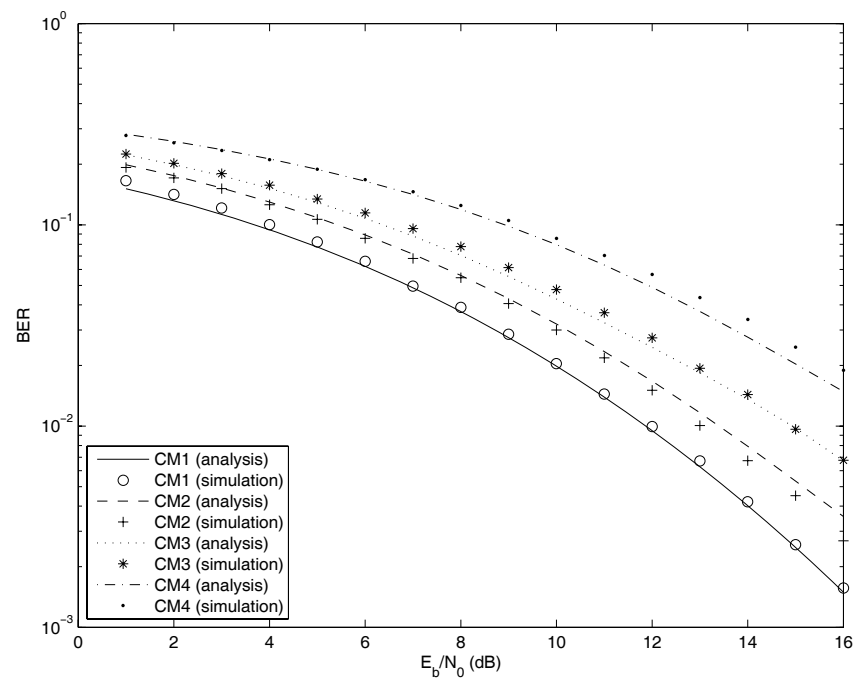

Fig. 3. BER performance of the proposed characteristic function (CF)-based analytical formula (11) for the IEEE 802.15.3a UWB channels CM1 4. The number of RAKE receiver fingers is 10 . The shadowing standard deviation $\sigma_{x}=6 \mathrm{~dB}$. The modulation index of PPM $\delta=1$ and the chip duration $T_{\mathrm{c}}=1 \mathrm{nsec}$.

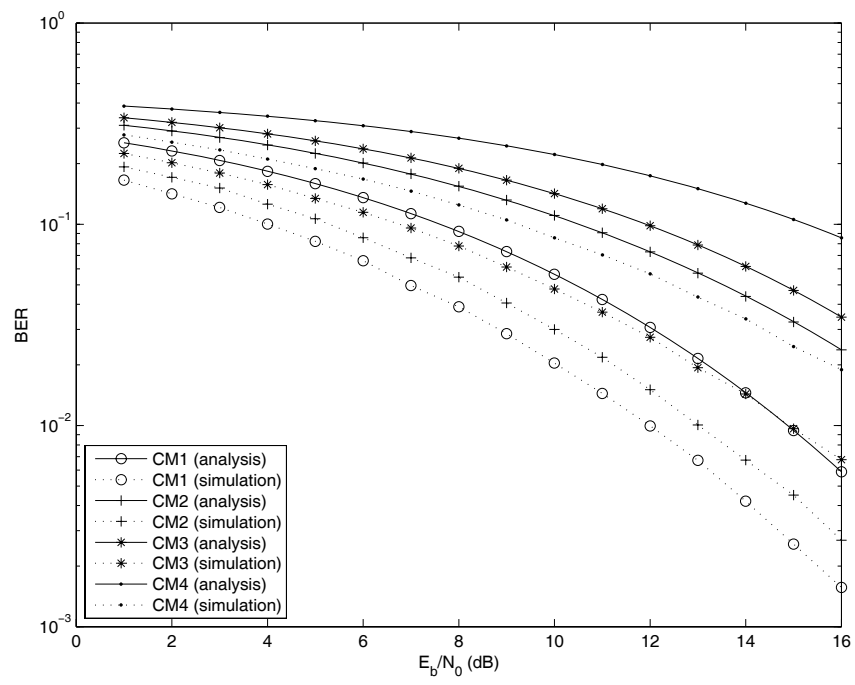

Fig. 4. BER performance of the moment generating function (MGF)-based analytical formula (12) for the IEEE 802.15.3a UWB channels CM1 4. The number of RAKE receiver fingers is 10 . The shadowing standard deviation $\sigma_{x}=6 \mathrm{~dB}$. The modulation index of PPM $\delta=1$ and the chip duration $T_{\mathrm{c}}=1 \mathrm{nsec}$.

deviation $\sigma_{x}=6 \mathrm{~dB}$. Surprisingly, one can see large errors between the analytical and simulation BER curves. Thus, the MGF-based BER formula (12) and the Craig's formula may not be adequate for BER analysis in the IEEE 802.15.3a UWB channel when shadowing is included.

Fig. 5 shows the PDF $f_{\tilde{\mathcal{E}}}(x)$ in the channel model CM1 for RAKE finger numbers $L=10 \sim 50$. In the low energy range, the curve of $L=10$ has the most probability mass; the curve of $L=20$ ranks second; the curve of $L=30$ ranks third; the curve of $L=40$ ranks fourth; and the curve of $L=50$ has the least probability mass. This phenomenon can explain why the case of $L=10$ has the worst BER performance compared to $L=20,30,40$, and 50 .

Fig. 6 shows the effect of various RAKE finger numbers 


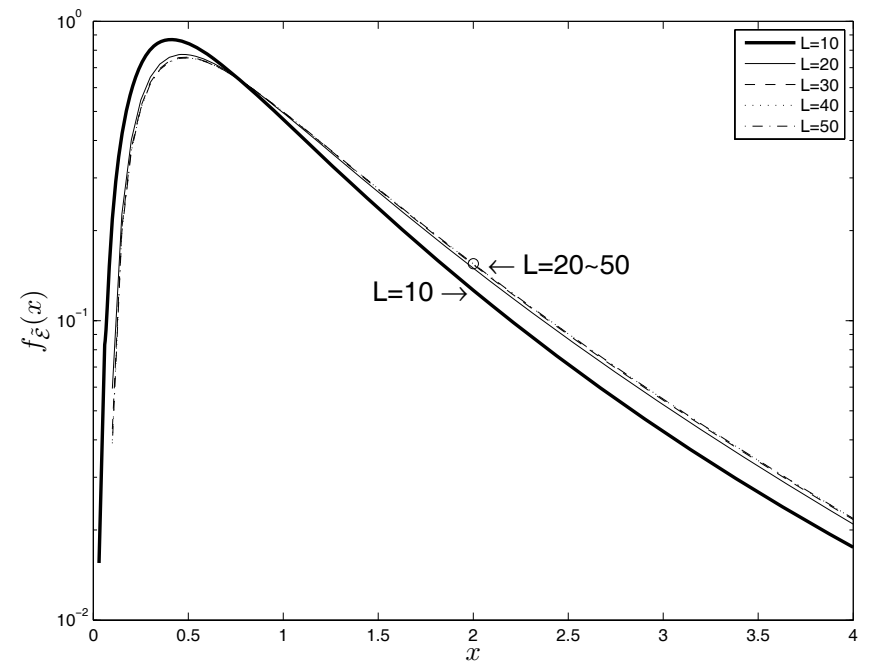

Fig. 5. The PDF $f_{\tilde{\mathcal{E}}}(x)$ of the received energy $\tilde{\mathcal{E}}$ of a RAKE receiver with number of fingers $L=10,20,30,40$, and 50 in the IEEE 802.15.3a UWB channel CM1.

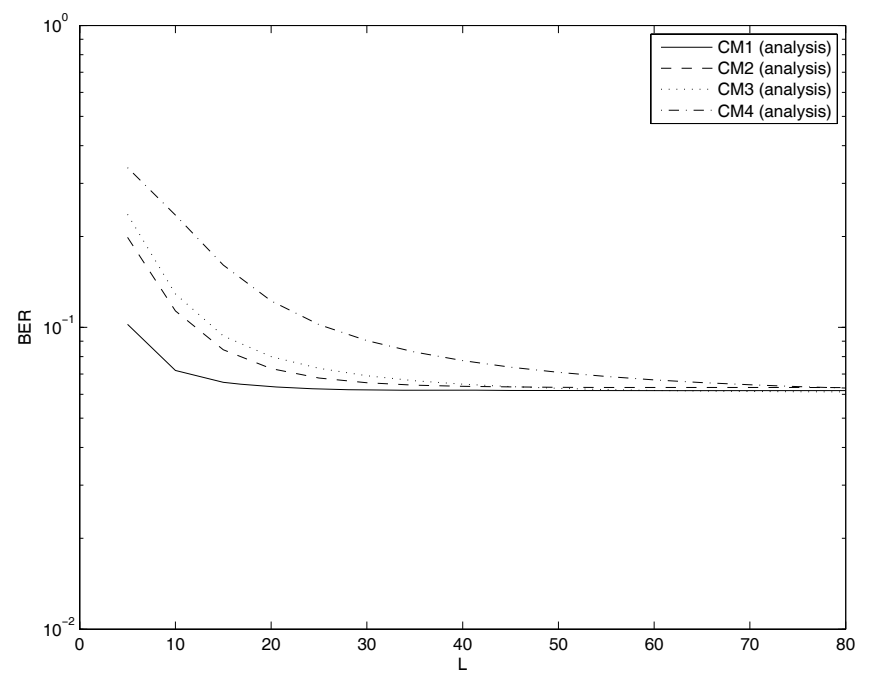

Fig. 6. BER v.s. the number of fingers of the RAKE receiver $(L)$ for $\mathrm{CM} 1 \sim 4$, where $E_{\mathrm{b}} / N_{0}=5 \mathrm{~dB}$.

on the BER performance of orthogonal binary signals (i.e., $\left.\rho_{r}=0\right)$ in the IEEE 802.15.3a CM1 4 channel models. We set $E_{\mathrm{b}} / N_{0}=5 \mathrm{~dB}$ and adopt the same parameters as in Fig. 3 . In general, the BER decreases as $L$ increases because more signal energy is collected at the RAKE receiver. For a large value of $L$, the BER curves become flat. This is because the RAKE receiver already captures most signal energy. Fig. 6 shows that the four channels actually give almost the same BER result (about $6 \times 10^{-2}$ ) when the number of fingers is increased up to 80 .

In the above results, we find that our proposed analytical method can save a significant amount of computer simulation time. For example, my computer spends 80 minutes to calculate the analytical BER in Fig. 1. However, the same computer needs 80 hours to produce the simulation BER. Note: My computer is equipped with $\operatorname{Intel}(\mathrm{R}) \operatorname{Pentium}(\mathrm{R}) 4$ CPU 2.40GHz and 1GB RAM.

\section{Conclusions}

In this work, we have derived the computable BER formula for a RAKE receiver in the complete IEEE 802.15.3a UWB channel models. We propose an approximation technique for the collected energy at an $L$-finger RAKE receiver. We find that the proposed BER computation method can save a significant amount of computer simulation time. Furthermore, we propose a CF-based BER formula to overcome the convergence problem of MGF-based BER formula when shadowing is included. The accuracy of the proposed technique is verified by simulations. Our results quantitatively indicate the effect of shadowing and RAKE finger numbers on BER performance in the IEEE 802.15.3a UWB channel. One interesting research topic that can be extended from this work is to apply this method to any multipath fading models with other general random arrival process for clusters and rays, such as the IEEE 802.15.4a channel.

\section{APPENDIX A \\ PROOF OF THEOREM 1}

Let $f_{G \mid T, t}(x)$ be the PDF of the path gain $G \triangleq \alpha_{k, l}$ arriving at time $t$ with a cluster that starting at time $T$. According to [15], it follows that $f_{G \mid T, t}(x)=\frac{1}{2}\left[f_{|G| \mid T, t}(x)+f_{|G| \mid T, t}(-x)\right]$ because the path gain is positive or negative with equal probability of 0.5 . Note that $f_{|G| \mid T, t}(x)$ is lognormally distributed, i.e., $f_{|G| \mid T, t}(x)=\frac{20 \exp \left[-\frac{1}{2 \sigma^{2}}\left(20 \log _{10} x-\mu_{T, t}\right)^{2}\right]}{\sqrt{2 \pi} \sigma x \ln 10}, x>0 ; 0$, otherwise. Thus, it follows that $20 \log _{10} G^{2}=2\left(20 \log _{10}|G|\right) \propto$ $\operatorname{Normal}\left(2 \mu_{T, t},(2 \sigma)^{2}\right)$. Then the PDF of the squared path gain arriving at time $t$ in a cluster starting at time $T$ can be written as $f_{T, t}(x)=\frac{10 \exp \left[-\frac{1}{2 \sigma^{2}}\left(10 \log _{10} x-\mu_{T, t}\right)^{2}\right]}{\sqrt{2 \pi} \sigma x \ln 10}, x>0 ; 0$, otherwise. Denote $\tilde{\mathcal{L}}_{T, t}(\nu)$ as the characteristic function of $f_{T, t}(x)$, then we can obtain (4).

\section{APPENDIX B \\ PROOF OF THEOREM 2}

As in [15], we define $\tilde{\psi}_{\nu}(T, L)=\int_{\max (a, T)}^{b}\left[1-\tilde{\mathcal{L}}_{T, t}(\nu)\right] d t$ for $T \leq b ; 0$ for $T>b$. Let $\tilde{J}(\nu, L)=\int_{0}^{a}\left[1-e^{-\lambda \tilde{\psi}_{\nu}(T, L)}\right] d T+$ $\int_{a}^{b}\left[1-\tilde{\mathcal{L}}_{T, T}(\nu) e^{-\lambda \tilde{\psi}_{\nu}(T, L)}\right] d T$. By setting $a=0$ and $b=$ $L T_{\mathrm{c}}$, we can transform the above equations to (5) and (6), respectively.

\section{REFERENCES}

[1] J. Foerster, et al., "Channel modeling sub-committee report final," IEEE P802.15 Wireless Personal Area Networks, P802.15-02/490r1-SG3a, Feb. 2003.

[2] J. Foerster and Q. Li, "UWB channel modeling contribution from intel," IEEE P802.15-02/279-SG3a, June 2002.

[3] D. Cassioli, M. Z. Win, and A. F. Molisch, "The ultra-wide bandwidth indoor channel: from statistical model to simulations," IEEE J. Sel. Areas Commun., vol. 20, no. 6, pp. 1247-1257, Aug. 2002.

[4] K. Eshima, Y. Hase, S. Oomori, F. Takahashi, and R. Kohno, "M-ary UWB system using Walsh codes," in Proc. IEEE Conf. Ultra Wideband Syst. and Technologies, Baltimore, MD, USA, May 2002, pp. 37-40.

[5] M. Hämäläinen, R. Tesi, and J. Iinatti, "On the UWB system performance studies in AWGN channel with interference in UMTS band," in Proc. IEEE Conf. Ultra Wideband Syst. and Technologies, Baltimore, MD, USA, May 2002, pp. 321-325.

[6] İ. Güvenç and H. Arslan, "Performance evaluation of UWB systems in the presence of timing jitter," in Proc. IEEE Conf. Ultra Wideband Syst. and Technologies, Virginia, USA, Nov. 2003, pp. 136-141. 
[7] T. Q. S. Quek and M. Z. Win, "Ultrawide bandwidth transmittedreference signaling," in Proc. IEEE Int. Conf. Commun., vol. 6, pp. 3409-3413, June 2004.

[8] J. A. Gubner and K. Hao, "A computable formula for the average biterror probability as a function of window size for the IEEE 802.15.3a UWB channel model," IEEE Trans. Microwave Theory Tech., vol. 54, no. 4, pp. 1762-1777, Apr. 2006.

[9] K. Hao and J. A. Gubner, "Performance measures and statistical quantities of rake receivers using maximal-ratio combining on the IEEE 802.15.3a UWB channel model," IEEE Trans. Wireless Commun., submitted for publication.

[10] W. P. Siriwongpairat, W. Su, and K. J. R. Liu, "Characterizing performance of multiband UWB systems using Poisson cluster arriving fading paths," in Proc. IEEE Workshop on Signal Process. Advances in Wireless Commun. (SPAWC), July 2005, pp. 264-268.

[11] L.-C. Wang, W.-C. Liu, and K.-J. Shieh, "On the performance of using multiple transmit and receive antennas in pulse-based ultrawideband systems," IEEE Trans. Wireless Commun., vol. 4, no. 6, pp. 2738-2750,
Nov. 2005.

[12] H. Liu, R. C. Qiu, and Z. Tian, "Error performance of pulse-based ultra-wideband MIMO systems over indoor wireless channels," IEEE Trans. Wireless Commun., vol. 4, no. 6, pp. 2939-2944, Nov. 2005.

[13] W.-D. Wu, C.-C. Lee, C.-H. Wang, and C.-C. Chao, "Signal-tointerference-plus-noise ratio analysis for direct-sequence ultra-wideband systems in generalized Saleh-Valenzuela channels," IEEE J. Sel. Topics Signal Process., vol. 1, no. 3, pp. 483-497, Oct. 2007.

[14] J. G. Proakis, Digital Communications, 4th ed. Boston: McGraw-Hill, 2001.

[15] K. Hao and J. A. Gubner, "The distribution of sums of path gains in the IEEE 802.15.3a UWB channel model," IEEE Trans. Wireless Commun., vol. 6, no. 3, pp. 811-816, Mar. 2007.

[16] P. J. Davis and P. Rabinowitz, Methods of Numerical Integration, 2nd ed. San Diege: Academic Press, Inc., 1984.

[17] [Online]. Available: http://www.efunda.com/math/num_integration/ num_int_gauss.cfm 